\title{
Gelatin as a chlorhexidine digluconate immobilizing agent
}

\begin{abstract}
Periodontal disease is an infectious-inflammatory disease that affects the supporting tissues of the teeth.This process results in inflammation within the supporting tissues of the teeth, progressive loss of attachment and bone loss and is characterized by periodontal pocket formation and/or recession of the gingiva. Chlorhexidine is a chemical agent widely used in dentistry, in the fight against periodontal disease and a wide range of infections, mainly thanks to its potent antimicrobial activity. Despite the excellent properties, chlorhexidine is an extremely hydrophilic drug, usually obtained in the aqueous solution form, making it hard to be incorporated into polymer controlled release systems. Successive lyophilization processes were inefficient for water removal. This work aimed to use gelatin as a chlorhexidine immobilizing agent. This procedure allowed the obtaining of a powder, which will be used as a drug controlled release film. The incorporation of chlorhexidine in a hydrophilic polymer matrix is already a well-established procedure. However, aiming the future preparation of a slower release device, we proposed the incorporation of the drug into a hydrophobic polymer matrix. To achieve this next purpose, it was necessary the immobilization of chlorhexidine in gelatin, which is reported here. More specifically, three gelatin and chlorhexidine systems were prepared. The characterizations were performed to understand the effect of different amounts of chlorhexidine on the obtaining of the system as a powder by lyophilization. Besides that, the capability of these systems to generate a microbiological response against two of the more significant microorganisms involved in the development of periodontal disease, Aggregatibacter actinomycetemcomitans (A.a), and Porphyromonas gingivalis (P.g), were evaluated as well. The results showed that the incorporation was carried out successfully. Also, the aspect of the obtained powder has a direct relation to the concentration of chlorhexidine. Regarding the microbiological test, the results show that all systems are capable of producing an efficient inhibition halo.
\end{abstract}

Keywords: controlled release of drugs, gelatin, chlorhexidine, periodontal disease
Volume I Issue 5 - 2017

\author{
Nathali Ricardo,' Fernando Gomes de \\ Souza,' Maíra do Prado, ${ }^{2}$ Maicon Ricardo \\ Zieberg Passini ${ }^{3}$ \\ 'Instituto de Macromoléculas, Universidade Federal do Rio de \\ Janeiro, Brazil \\ ${ }^{2}$ Universidade Veiga de Almeida, Centro de Saúde, Brazil \\ ${ }^{3}$ Faculdade de Odontologia de Piracicaba, Departamento de \\ Odontologia Restauradora, Universidade Estadual de Campinas, \\ Brazil
}

Correspondence: Fernando G de Souza, Professor, Universidade Federal do Rio de Janeiro, Cidade Universitária. Av. Horácio Macedo, 2.030. Centro de Tecnologia-Prédio do Bloco J CEP 2194I-598-Rio de Janeiro-RJ-Brasil,Tel 0055213938 7766, Email fgsj@ufrj.br

Received: October 28, 2017 | Published: November 29, 2017
Abbreviations: CRS, controlled drug release system; FTIR, fourier-transform infrared spectroscopy; TGA, thermogravimetric analysis; CHX, chlorhexidine G; DSC, differential scanning calorimeter; A.a, aggregatibacter actinomycetemcomitans; P.g, porphyromonas gingivalis

\section{Introduction}

Periodontal diseases are a diversified group of clinical entities, described as pathologies of a multifactorial nature, in which the induction of an inflammatory process that destroys the fixation apparatus, loss of the alveolar bone and, if untreated, in the loss of the dental element. Among the wide variety of drugs used in the treatment of periodontal disease, chlorhexidine demonstrated to be a highly active chemical agent for microbial control. Chlorhexidine possesses the molecular formula $\mathrm{C}_{22} \mathrm{H}_{30} \mathrm{Cl}_{2} \mathrm{~N}_{10}$, a molar mass equal to $505.4 \mathrm{~g} / \mathrm{mol}$, the density of $1.06 \mathrm{~g} / \mathrm{cm}^{3}$ and a boiling point of $134^{\circ} \mathrm{C} .{ }^{1}$ The antimicrobial effect of chlorhexidine is due to the attraction and adsorption of the chlorhexidine cationic molecules on the surface of the microorganism's cells. ${ }^{2}$ This interaction promotes the alteration of the cell membrane permeability, resulting in the loss of intracellular components and the osmotic imbalance of the cell., ${ }^{3,4}$ Its sizeable microbial spectrum, persistence, and low toxicity, ${ }^{5,6}$ promoted the use of this substance in several areas related to the health. Also, the most widely used form of chlorhexidine is the $0.12 \%$ chlorhexidine digluconate solution. ${ }^{7}$ Despite presenting excellent antimicrobial activity, the effectiveness of conventional treatment of oral diseases is often limited due to reduced retention of formulations applied in the oral cavity. The salivary flow, the swallowing reflex, chewing and speaking may affect the dilution or displacement of a pharmaceutical formulation and can lead to a rapid decline in the concentration of the active ingredients to sub-therapeutic levels. Thus, there is a need for the development of drug delivery systems that could confer a better bio-availability of active components and a more significant retention of this active substance in the place where it should act while allowing a reduction in the frequency of administration and dosing. ${ }^{8}$ By definition, the Controlled Drug Release System (CRS) is an administration system designed to prolong the release time of the drug in the body, maintain its plasma concentration and control the temporal and spatial location of the molecules in vivo, by applying biological and chemical principles. Apart from this concept, there are already existing systems of local release of antibiotics or antiseptic solutions that are being used to restrict the action of the drug to the patient site and to minimize the systemic effects. The PerioChip ${ }^{\circledR}$ is a classic example of this type of apparatus. This device contains $2.5 \mathrm{mg}$ of chlorhexidine gelatinous matrix. The literature showed that one of the major problems with this device is the initial burst of the unbound chlorhexidine, which is released in the first 48 hours, during what more than $40 \%$ of the chlorhexidine is released. ${ }^{9}$ 
The development of a device able to quickly reach the minimum CHX concentration besides keep as long as possible the drug release is the primary goal for better treatments. In this context, the use of polyesters deserves special mention because they have many applications in biomedicine due to their slow degradation, high permeability to many drugs and low toxicity. Thus, the failure to encapsulate chlorhexidine $e^{10,11}$ to the polymer matrix of interest via emulsion due to incompatibility between both components is a stimulus to the development of a powder formulation so that the drug could be incorporated into the polymer matrix and thus, a more efficient controlled release system could be developed for use in the oral cavity. To achieve this goal, gelatin, a natural polymer produced from collagen hydrolysates or the polysaccharide fraction of seaweed was used as a Chlorhexidine immobilizer. ${ }^{12-16}$ Differently from other studies, which were focused on the use gelatin as the primary polymer matrix, the present study aims to obtain a powder of chlorhexidine in a fast and cheap way. Aiming this, gelatin was used as an immobilizing agent. Thus, the obtained gelatine-chlorhexidine powder would easily be incorporated into another polymer matrix by a simple melt mixing method, making easier the obtaining of a device for the even slower release of the drug and a prolonged local time of action.

\section{Materials and methods}

\section{Materials}

Gelatin powder, from SYNTH and Chlorhexidine gluconate (CHX) 20\%(w/v), from MIL FORMULAS, Brasil. All the reactants were used as received.

\section{Methods}

Gelatin-Chlorhexidine mixtures: The mixtures were prepared using the same amount of gelatin $(10 \mathrm{~g})$. To all systems, the final volume of the obtained solution was equal to $50 \mathrm{ml}$. Soon afterward the complete dissolution of the gelatin, the following volumes of chlorhexidine was added to each system: $15 \mathrm{~mL}$ ( $3 \mathrm{~g}$ of $\mathrm{CHX}$ ), $25 \mathrm{~mL}$ ( $5 \mathrm{~g}$ of CHX) and $50 \mathrm{~mL}(10 \mathrm{~g}$ of CHX). These systems were named as $3 \mathrm{G}, 5 \mathrm{G}$, and $10 \mathrm{G}$, respectively. The amounts of $\mathrm{CHX}$ in the systems $3 \mathrm{G}, 5 \mathrm{G}$ and $10 \mathrm{G}$ were equal to $23,1 \%, 33,3 \%$, and $50 \%$, respectively. The mixtures were poured into beakers for $2 \mathrm{~min}$, and subsequently, the obtained materials were poured into 4 glass plates. These plates were placed in the refrigerator for 24hours. After 24hours in the refrigerator, the Gelatin-Chlorhexidine mixtures were passed through a bath in liquid nitrogen and lyophilized to remove excess water in a Liotop L101 freeze dryer. The $3 \mathrm{G}$ ( $3 \mathrm{~g}$ of CHX), 5G (5g of CHX), and 10G (10g of CHX) samples were lyophilized for $30 \mathrm{~h}, 24 \mathrm{~h}, 18 \mathrm{~h}$, respectively. The different lyophilization times had the objective of removing the water until reaching constant mass. Once the excess water was removed, the samples were ground with the aid of an IKA A11 crusher and then sieved in a $100 \mu \mathrm{m}$ aperture particle size sieve until a fine powder was obtained for each system.

The microbiological test was performed to establish a comparison with the future polymeric devices developed. The test is based on the formation of an inhibition zone demonstrating that antimicrobial activity of the samples of gelatin-chlorhexidine in a test of agar diffusion front of the following microorganisms: Aggregatibacter actynomicetemcomitans (A.a) and Porphyromonas gingivalis (P.g). The microorganisms were selected taking into consideration that A.a and P.g are among the leading etiological agents of periodontal disease. Pure gelatin and $0.12 \%$ Perioxidine gel (Lacer.) were used as the control group.

\section{Results and discussion}

Aiming to standardize all the parameters, the obtained materials were subjected to different lyophilization times to eliminate the excess of water. The difference in these lyophilization times is related to the decrease of the intermolecular forces. Thus, the $10 \mathrm{G}$ group needed the smaller lyophilization time. A linear correlation test was performed to prove the relationship between the CHX concentration and the time of lyophilization, and the obtained value was equal to- 0.99 which indicates that there is a high correlation between the measured parameters. This result is shown in Table 1.

Figure 1 shows the TGA of the samples. Obtained data allowed inferring that the CHX solution lost about $79 \%$ of its mass up to $117^{\circ} \mathrm{C}$, proving that the drug content indicated by the manufacturer is correct, being around $20 \%$. Besides that, the drug remains stable up to $160^{\circ} \mathrm{C}$. Soon afterward, the $\mathrm{CHX}$ presents two degradation events. The first finished at $244^{\circ} \mathrm{C}$, and the second at $497^{\circ} \mathrm{C}$. The gelatin lost $\mathrm{H}_{2} \mathrm{O}$ up to $145^{\circ} \mathrm{C}$, leaving a $40 \%$ residue of gelatin which starts to degrade at $281^{\circ} \mathrm{C}$. In turn, the systems contained $\mathrm{CHX}$ in gelatin presented a common thermal behavior: as samples were thermally stable up to $130^{\circ} \mathrm{C}$. Then, they suffered 2 degradation steps. $3 \mathrm{G}, 5 \mathrm{G}$, and $10 \mathrm{G}$ samples presented their first maximum degradation rate at $202^{\circ} \mathrm{C}, 206^{\circ} \mathrm{C}$, and $205^{\circ} \mathrm{C}$, respectively. The same samples presented their second maximum degradation rate at $348^{\circ} \mathrm{C}, 346^{\circ} \mathrm{C}$, and $346^{\circ} \mathrm{C}$, respectively. Therefore, the temperature used in the preparation of the samples did not affect the CHX, and the mixing process can be considered secure to the drug.

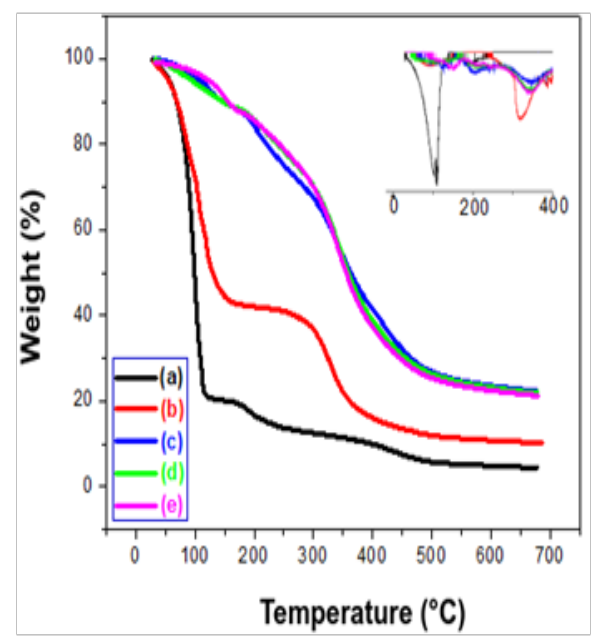

Figure I TGA of the Chlorhexidine (A), gelatin (B) and 3G (C), 5G (D) and IOG (E) samples. The inset shows the derivative weight of the samples.

Table I Relationship between the amount of distilled water and the lyophilization time of each sample

\begin{tabular}{lll} 
Sample & $\mathbf{C H X}(\%)$ & Lyophilization time \\
\hline $3 G$ & 23.1 & 30 \\
$5 G$ & 33.3 & 24 \\
I0G & 50 & 18 \\
Correlation & & -0.99 \\
\hline
\end{tabular}


Figure 2 shows the DSC of the samples. CHX presents an endothermic event around $106^{\circ} \mathrm{C}$. This event is attributed to the evaporation of the water present. There is another endothermic event smaller, which took place around $186^{\circ} \mathrm{C}$. This is due to the fusion of chlorhexidine..$^{17}$ Pure gelatin has an endothermic peak ranging from $46^{\circ} \mathrm{C}$ to $160^{\circ} \mathrm{C}$. This event occurs due to the loss of water and it is longer than the CHX one due to hydrogen bonds between the gelatin and the water. Systems containing the lyophilized mixture of $\mathrm{CHX}$ and gelatin were not influenced by the presence of water since they were analyzed immediately after lyophilization. These systems presented endothermic peaks between $155^{\circ} \mathrm{C}$ and $180^{\circ} \mathrm{C}$, which are related to the melting of $\mathrm{CHX}$.

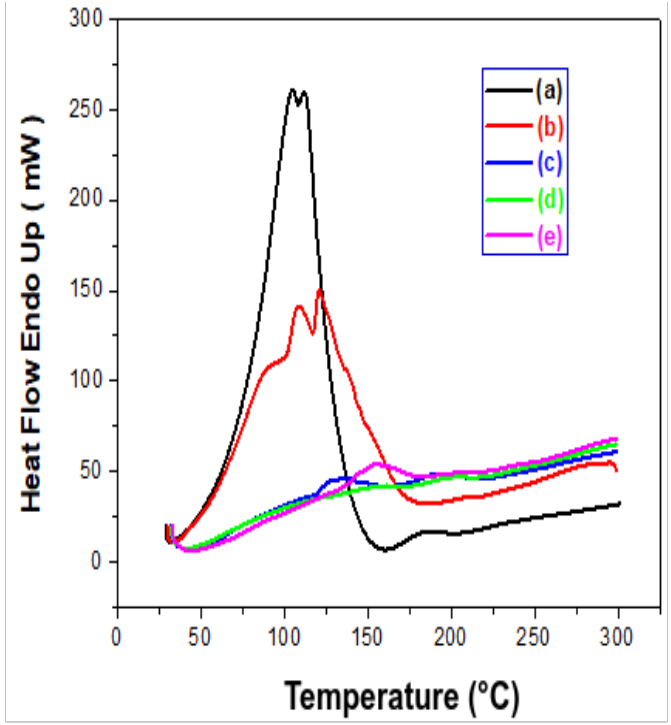

Figure 2 DSC Chlorhexidine (A), gelatin (B) and 3G (C), 5G (D) and IOG (E) samples.

The Fourier Transform Infrared Spectroscopy technique was used as a characterization method to prove the incorporation of the drug into the gelatin. Figure 3 shows a comparison between pure gelatin spectra and samples containing CHX. All the samples containing the drug presented the $\mathrm{N}-\mathrm{H}$ characteristic band of the $\mathrm{CHX}$ amine at $1640 \mathrm{~cm}^{-1}$. This band was used as a reference for the present amount of CHX. As one can see in the inset of Figure 3, as higher the amount of CHX, as intense the transmittance of the CHX amine peak. The FTIR band centered at $1640 \mathrm{~cm}^{-1}$ was used to correlate the chlorhexidine concentration present in these samples with the transmittance. The obtained results allowed inferring that the higher the chlorhexidine concentration, the greater the transmittance of the $1640 \mathrm{~cm}^{-1}$. The corresponding coefficient of determination $\left(\mathrm{R}^{2}\right)$ was equal to 0.9588 . Therefore, the increasing amount of CHX in the materials was proved.

Table 1 shows the average results obtained using triplicates of the microbiological test for A.a, and P.g. The Figures $4 \&$ Figure 5 showed the inhibition halo of the tested groups, from which data of Table 2 was obtained. The results allowed inferring that each one of the developed gelatine-chlorhexidine systems produced more magnificent inhibition halos than the commercial formulation. Moreover, it is noted that the systems produce higher antimicrobial activity against P. g. Therefore, the obtained results proved the encapsulation of the CHX in the gelatin, which can be the basis for the preparation of cheaper drug delivery systems for the treatment of several dentistry diseases.

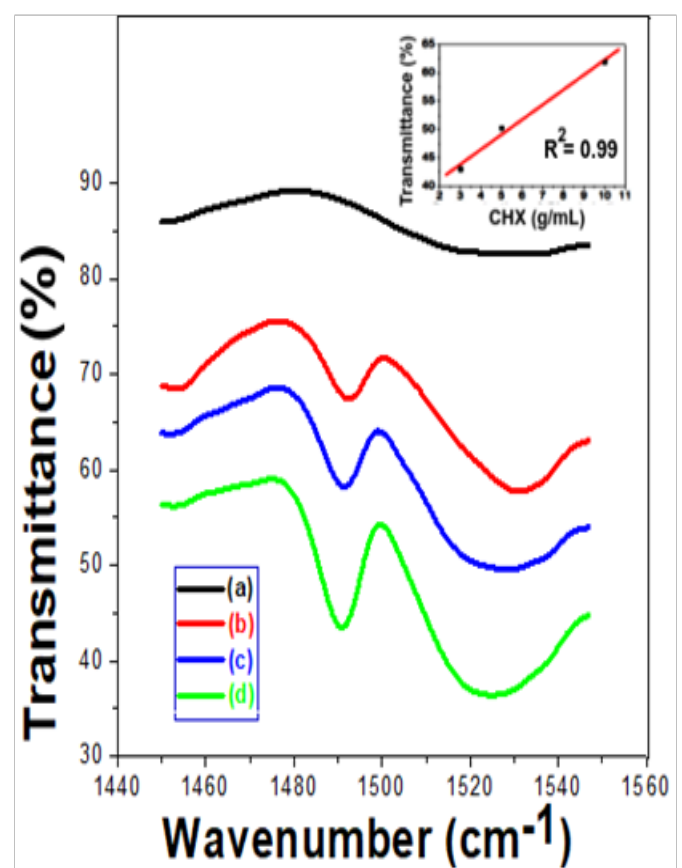

Figure 3: FTIR spectra of gelatin (a) and 3G (b), 5G (c) and IOG (d) samples. The inset shows the $\mathrm{R} 2$ between transmittance and the $\mathrm{CHX}$ concentration.

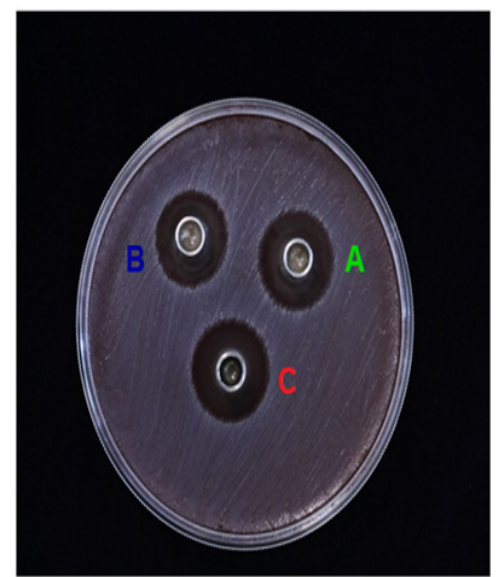

Figure 4: Microbiological test with evidence of inhibition halos against $A . a$ microorganisms. Tested samples: 3G (A), 5G (B) and I0G (C).

Table 2 Inhibition halo test performed in triplicate for the A.a and P.g. microorganisms

\begin{tabular}{lll} 
Samples & A.a $(\mathbf{m m})$ & P.g $(\mathbf{m m})$ \\
\hline 3G & $21.7 \pm 4.7$ & $38.1 \pm 0.2$ \\
$5 G$ & $20.3 \pm 1.1$ & $37.3 \pm 0.2$ \\
IOG & $21.1 \pm 0.7$ & $36.4 \pm 0.9$ \\
Pure Gelatin & $0.0 \pm 0.0$ & $0.0 \pm 0.0$ \\
Perioxidine & $15.0 \pm 0.0$ & $32.0 \pm 0.0$
\end{tabular}




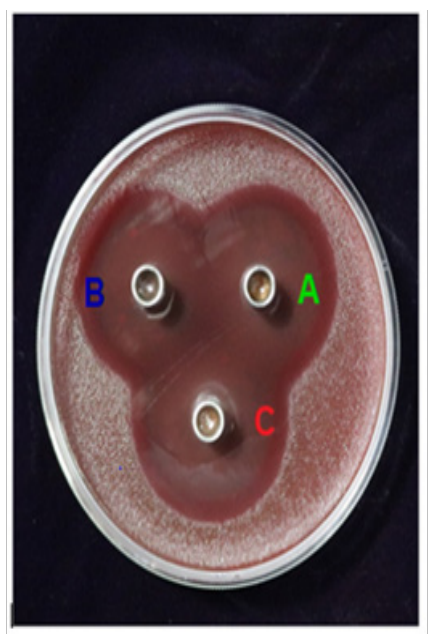

Figure 5: Microbiological test with evidence of inhibition halos against P.g microorganisms. Tested samples: 3G (A), 5G (B) and I0G (C).

\section{Conclusion}

Gelatin can be used as the hydrophilic drug immobilizing agent. Besides, unlike other controlled release devices for CHX based on polymer systems, the material presented here were prepared without the use of organic solvents, which would be a way of making the release devices cheaper and more readily available for the population.

\section{Acknowledgements}

The authors wish to acknowledge development institutions that have made this work possible: CNPq, CAPES, FINEP e a FAPERJ. Coleção de Microrganismos de Referência em Vigilância Sanitária CMRVS Fundação Oswaldo Cruz - FIOCRUZ-Instituto Nacional de Controle de Qualidade em Saúde - INCQS-Lab. de Microrganismos de Referência

\section{Conflict of interest}

The author wish to state that there is no conflict of interest.

\section{References}

1. Franco CF, Pataro AL, E Souza LCR, et al. In vitro effects of a chlorhexidine controlled delivery system. Artificial Organs. 2003;27(5):486-491.

2. Gaetti-Jardim EC, Marqueti AC, Faverani LP. Antimicrobial resistance of aerobes and facultative anaerobes isolated from the oral cavity. $J$ Appl Oral Sci. 2010;18(6):551-559.

3. Ferraz CC, Gomes BP, Zaia AA, et al. In vitro assessment of the antimicrobial action and the mechanical ability of chlorhexidine gel as an endodontic irrigant. $J$ Endod. 2001;27(7):452-455.
4. Goncalves LS, Rodrigues RCV, Andrade Junior CV, et al. The effect of sodium hypochlorite and chlorhexidine as irrigant solutions for root canal disinfection: a systematic review of clinical trials. $J$ Endod. 2016;42(4):527-532.

5. Afra SM, Modaresi2 F. The Use of synergistically antiplaque nanoparticles in treating dental caries. Journal of Dental Health Oral Disorders \& Therapy. 2017;6(5):1-6.

6. Shahani MN, Subba Reddy VV. Comparison of antimicrobial substantivity of root canal irrigants in instrumented root canals up to $72 \mathrm{~h}$ : an in vitro study. Journal of the Indian Society of Pedodontics and Preventive Dentistry. 2011;29(1):28-33.

7. Swadas M, Dave B, Vyas SM, et al. Evaluation and comparison of the antibacterial activity against streptococcus mutans of grape seed extract at different concentrations with chlorhexidine gluconate: an in vitro study. Int J Clin Pediatr Dent. 2016;9(3):181-185.

8. Yadiki JV, Jampanapalli SR, Konda S, et al. Comparative evaluation of the antimicrobial properties of glass ionomer cements with and without chlorhexidine gluconate. Int J Clin Pediatr Dent. 2016;9(2):99-103.

9. Puri K, Dodwad V, Bhat K, et al. Effect of controlled-release PeriochipTM on clinical and microbiological parameters in patients of chronic periodontitis. J Indian Soc Periodontol. 2013;17(5):605-611.

10. Barrios R, Ferrer-Luque CM, Arias-Moliz MT, et al. Antimicrobial substantivity of alexidine and chlorhexidine in dentin. $J$ Endod. 2013;39(11):1413-1415.

11. Zhou Y, Hu K, Guo Z, et al. PLLA microcapsules combined with silver nanoparticles and chlorhexidine acetate showing improved antibacterial effect. Mater Sci Eng C Mater Biol Appl. 2017;78:349-353.

12. Abdelaziz Ghanemi. Pharmaceutical forms preparation and drugs prescription: building an international system to meet the cultural aspects. Pharmacy \& Pharmacology International Journal. 2016;4(1):1-2.

13. Park K. Controlled drug delivery systems: Past forward and future back. J Control Release. 2014;190:3-8.

14. Sahoo N, Sahoo RK, Biswas N, et al. Recent advancement of gelatin nanoparticles in drug and vaccine delivery. Int J Biol Macromol. $2015 ; 81: 317-331$.

15. Santoro M, Tatara AM, Mikos AG. Gelatin carriers for drug and cell delivery in tissue engineering. J Controll Release. 2014;190:210-218.

16. Treesuppharat W, Rojanapanthu P, Siangsanoh C, et al. Synthesis and characterization of bacterial cellulose and gelatin-based hydrogel composites for drug-delivery systems. Biotechnol Rep (Amst). 2017;15:8491.

17. Souza-Filho FJ de, Soares A de J, Vianna ME, et al. Antimicrobial effect and $\mathrm{pH}$ of chlorhexidine gel and calcium hydroxide alone and associated with other materials. Braz Dent J. 2008;19(1):28-33. 